\title{
Modified shock index as a predictor of in-hospital outcome in cases of st-segment elevation myocardial infarction treated with primary percutaneous coronary intervention
}

Volume 7 Issue 4 - 2016

\section{Introduction}

In spite of great advances in diagnosis and management, ST segment elevation myocardial infarction (STEMI), it remains a major public health problem in the industrialized world and is on the rise in developing countries. ${ }^{1,2}$ Primary percutaneous coronary intervention (PPCI) is now classified as class I indication in STEMI in the guidelines of the European Society of Cardiology (ESC). ${ }^{3}$ Risk stratification for patients with STEMI is very important to identify those patients who deserve advanced measures. This is done through integration of various patient characteristics into a semi-quantitative score that can convey an overall estimate of a patient's prognosis. ${ }^{4}$ At present, several systems of risk stratification such as Thrombolysis in Myocardial Infarction (TIMI), Global Register Acute Coronary Events (GRACE), CADILLAC risk score, and EuroHeart risk score are used, but the sophisticated calculation usually makes them inconvenient to operate at bedside in daily clinical practice. ${ }^{5-8}$ The concept of shock index, defined as the ratio of heart rate and systolic blood pressure, first introduced by Allgower et al, in 1967 as a simple and effective means for gauging the degree of hypovolemia in hemorrhagic and infectious shock states. ${ }^{9}$ Subsequently, experimental and clinical studies demonstrated that SI was inversely related to physiologic parameters, such as cardiac index, stroke volume, left ventricular stroke work, and mean arterial pressure. ${ }^{10}$

A new index, modified shock index (MSI), is created by Ye-cheng et al., ${ }^{16}$ as the ratio of HR and mean arterial pressure (MAP). He noticed that SI uses only systolic blood pressure, but diastolic blood pressure (DBP) is also of undeniable importance when determining patient's clinical severity. Hence he incorporated diastolic blood pressure and developed the modified shock index (MSI). ${ }^{16}$

\section{Subjects and methods}

This study was carried prospectively and included 216 patients who presented to CCU of Cardiology department, Zagazig university hospitals with acute STEMI who were treated with PPCI (from 1/2014 until 8/2016), with A time window for doing PPCI of 12 hours from onset of maximum chest pain. Extended time window to 18 hours from onset of maximum chest pain was used for patients presented with cardiogenic shock. We excluded the patients who, received fibrinolytic therapy, presented more than 12 hours from the onset of chest pain except for whom presented with cardiogenic shock, died at ER or before doing PPCI and presented by acute hemorrhage, acute hypovolemia, severe sepsis, severe trauma, or suspicion of pulmonary embolism.

Patients who met the inclusion criteria were subjected to thorough history taking, Physical examination with special emphasis on both

\author{
Mohammad Gouda, Aly M Saad, Mohammad \\ MAl- Daydamony
}

Cardiology Department, Faculty of Medicine, Zagazig University, Egypt

Correspondence: Mohammad Gouda, Cardiology Department, Zagazig University, Egypt, Tel 0201019693393, Email cardioman77@yahoo.com

Received: November 23, 2016 | Published: December I5, 2016

Blood pressure and pulse that were measured accurately in the supine position with $>2$ readings were taken with $1 \mathrm{~min}$ interval, and averaged. Admission SI was calculated for every patient that equals (admission $\mathrm{HR}$ / admission SBP). Modified Admission SI was calculated for every patient that equals (admission $\mathrm{HR} /$ mean admission $\mathrm{BP}$, here $\mathrm{MBP}=$ $(\mathrm{DBP} 2)+\mathrm{SBP} / 3)$. The optimal cut-off values of SI, and MSI were based on optimizing the sum of sensitivity and specificity by receiver operating characteristic curve analysis, which predicted in-hospital major adverse cardiovascular events.

The patients were divided into 4 groups:

Group A: This included patients with admission shock index $<$ cut off.

Group B: This included patients with admission shock index $>$ cut off.

Group C: This included patients with modified admission shock index $<$ cut off.

Group D: This included patients with modified admission shock index $>$ cut off.

The TIMI risk score was calculated for each patient using the variables obtained at admission according to the published criteria 5. Killip's classification, Chest examination: with emphasis on detecting pulmonary edema or presence of pulmonary congestion, Cardiac examination: with emphasis on detecting murmurs suspecting presence of mechanical complications. 12-lead surface ECG on first contact with patient then every 6hours and culprit artery will be identified. Laboratory investigations: Cardiac enzymes, $\mathrm{CBC}$-kidney function -blood sugar, Liver function and coagulation profile in selected patients. Coronary angiography and PPCI was performed 
by a 24-hours on-call interventional team, according to standard clinical practice from published guidelines. Coronary flow after revascularization, we used the Thrombolysis in Myocardial Infarction (TIMI) flow classification (TIMI $0,1,2,3){ }^{21}$ Patients then were admitted to our $\mathrm{CCU}$ for at least 48 hours to continue anti-ischemic treatment and where an ECG was performed immediately after and 90minutes after the PPCI to monitor ST segment resolution with chest pain resolution.

The primary endpoint was all-cause mortality during hospitalization. The secondary endpoint included major adverse cardiovascular events (MACE) which were composite of mortality, re-infarction, life-threatening arrhythmias, cardiogenic shock, stroke, heart failure, bleeding, and recurrent myocardial ischemia. (Were assessed and compared for each parameter ASA, MSA \& TIMI and according to that we will compare the three parameters with each other \& then we will calculate specificity and sensitivity for ASA \& MSA).

\section{Statistical analysis}

The baseline characteristics presented with mean + SD or median and interquartile range for continuous variables were compared by 1-way analysis of variance and Bonferroni correction if the data were normal distribution, otherwise by Wilcoxon signed rank test. Categorical variables presented as percentage were compared by the Pearson chi-square test. Cumulative survival and MACE curves were constructed with the Kaplan-Meier method. Log-rank tests were used to compare the curves of groups. Multivariate Cox proportional hazard regression models were performed to identify whether there was an association between SI, MSI and the in-hospital outcomes, and the models were corrected for age, gender, and other clinical parameters. The adjusted hazard ratios with their respective 95\% confidence intervals (CIs) for each group were calculated. The discriminatory capacity of SI for predicting in-hospital all-cause mortality was assessed using the area under the receiver operating characteristic curve. All statistical tests were 2-tailed, and $\mathrm{p}$ values were statistically significant at $<0.05$. All statistical analyses were carried out using the SPSS statistical software, version 20.0.

\section{Results}

The baseline Personal characteristics and risk factors among all study population are summarized in Table 1. Based on optimizing the sum of sensitivity and specificity by receiver operating characteristic curve analysis, the optimal cutoff value of ASI for predicting inhospital all-cause mortality \& MACE was 0.69 , and the sensitivity and specificity were $64.3 \%$ and $64.4 \%$, respectively. With this threshold, 119 patients had normal admission SI $(<0.69)$ and 97 patients had elevated admission SI $(>0.69)$. In regard to MSI, the optimal cutoff value of MSI for predicting in-hospital all-cause mortality\& MACE was 0.91 , and the sensitivity and specificity were $80.0 \%$ and $56.2 \%$, respectively. With this threshold, 96 patients had normal modified admission SI $(<0.91)$ and 120 patients had elevated modified admission SI $(>0.91)$.

\section{Admission shock index}

According to our study, there was highly statistically significant increase in age, PTD, HR, MSI, TIMI risk score, and Killip class IIIV (all $p<0.001)$, statistically significant increase in HTN $(p=0.009)$, and pre-infarction angina ( $p=0.02)$, and highly statistically significant decrease in SBP and DPB (all $p<0.001$ ) in patients with high ASI compared to patients with normal ASI. There was no statistically significant increase in cardiac enzymes $(p=0.13)$, DM $(p=0.17)$, history of IHD ( $p=0.68)$, smoking $(\mathrm{P}=0.75)$, positive family history of premature $\operatorname{CAD}(p=0.63)$, or history of stroke $(p=0.79)$, and there was no statistically significant decrease in $\mathrm{EF}(p=0.22)$, weight $(p=0.41)$ , history of previous procedures PCI or CABG $(p=0.29)$, or sinus rhythm $(p=0.96)$ in patients with high ASI compared to patients with normal ASI.

According to in-hospital MACE and mortality our study demonstrated statistically significant increase in cardiogenic shock $(p=0.002)$, and fatal arrhythmia $(p=0.027)$, and highly statistically significant increase in HF $(p=0.001)$, arrest $(p<0.001)$, and mortality $(p<0.001)$, while there was no statistically significant increase in reinfarction $(p=0.26)$, bleeding $(p=0.27)$, or stroke $(p=0.11)$, and no statistically significant decrease in non-fatal arrhythmia $(p=0.068)$ in patients with high ASI compared to patients with normal ASI (Table 2, Figure 1).

\section{Modified admission shock index}

According to our study there was highly statistically significant increase in age, PTD, HR, ASI, and TIMI risk score (all $p<0.001$ ) and statistically significant increase in Killip class II-IV $(p=0.014)$, while there was highly statistically significant decrease in SBP, and DPB (all $p<0.001)$ and statistically significant decrease in $\mathrm{EF}(p=0.004)$ in patients with high MSI compared to patients with normal MSI. There was no statistically significant increase in cardiac enzymes $(p=0.859)$ , DM ( $p=0.38)$, HTN $(p=0.51)$, history of IHD $(p=0.65)$, history of previous PCI $(p=0.27)$, positive family history of premature CAD $(p=0.09)$, pre-infarction angina $(p=0.19)$, or sinus rhythm $(p=0.45)$, and no statistically significant decrease in smoking $(p=0.87)$, or history of stroke $(p=0.78)$ in patients with high MSI compared to patients with normal MSI. According to in-hospital MACE and mortality our study demonstrated highly statistically significant increase in $\operatorname{HF}(p<0.001)$, and there was statistically significant increase in cardiogenic shock $(p=0.019)$, fatal arrhythmia $(p=0.007)$, bleeding $(p=0.026)$, arrest $(p=0.005)$, and mortality $(p=0.008)$, while there was no statistically significant increase in re-infarction $(p=0.37)$, non-fatal arrhythmia $(p=0.43)$, or stroke $(p=0.2)$ in patients with high MSI compared to patients with normal MSI (Table 2, Figure 1).

\section{TIMI risk score}

According to our study there was highly statistically significant increase in HF $(p<0.001)$, and there was statistically significant increase in cardiogenic shock $(p=0.014)$, non-fatal arrhythmia $(p=0.014)$, bleeding $(p=0.008)$, arrest $(p=0.001)$, and mortality $(p=0.006)$, while There was no statistically significant increase in reinfarction $(p=0.281)$, fatal arrhythmia $(p=0.068)$, or stroke $(p=0.127)$ in patients with high TIMIrs compared to patients with low TIMIrs (Table 2, Figure 1).

\section{Multivariate Cox regression analysis}

After adjusting for age, gender, histories of cardiovascular diseases and admission variables by univariate analysis, significant factors analyzed by Multivariate cox regression analysis to predict the independent factors for in-hospital mortality and MACE and revealed:

1. For mortality (primary end point): MSI was the strongest predictor of in-hospital mortality (OR 5.599, 95\% CI 4.128-8.15, $p=0.015)$. Other predictors were ASI (OR 2.87, 95\% CI 1.083.484, $p=0.04)$, TIMIrs (OR 1.238, 95\% CI 1.11-2.87, $p=0.021$ ), age (OR 1.079, 95\% CI 1.009-1.153, $p=0.025)$, and EF (OR $0.822,95 \%$ CI $0.741-0.911, p<0.001)$. 
2. For MACE (secondary end point): MSI was the strongest predictor of in-hospital MACE (OR 11.506, 95\% CI 10.5421.5664, $p=0.022)$. Other predictors were ASI (OR 1.21, 95\% CI 1.05-3.54, $p=0.012$ ), TIMIrs (OR 1.575, 95\% CI 1.094-2.269, $p=0.015)$, and EF (OR 0.879, 95\% CI 0.840-0.921, $p<0.001)$ (Table 3).

Table I Baseline characteristics and risk factors of the study population

\section{Correlations between study parameters}

Our study demonstrated significant positive correlation between ASI and modified SI and TIMIrs and also between TIMI and age modified SI but significant negative correlation between EF ASI, TIMI and Modified SI (Table 4, Figure 2-4).

\begin{tabular}{|c|c|c|c|c|c|c|c|c|}
\hline \multicolumn{2}{|l|}{ Variable } & $\begin{array}{l}\text { All Patients } \\
(n=2 \mid 6)\end{array}$ & $\begin{array}{l}\text { ASI > } 0.69 \\
(n=97)\end{array}$ & $\begin{array}{l}\text { ASI <0.69 } \\
(n=1 \mid 9)\end{array}$ & P-value & $\begin{array}{l}M S I>0.91 \\
(n=\mid 20)\end{array}$ & $\begin{array}{l}M S I<0.9 I \\
(n=96)\end{array}$ & P-value \\
\hline \multicolumn{2}{|l|}{ Age (year) } & $55.46+10.95$ & $58.72+10.60$ & $52.81+10.54$ & $0.00 * *$ & $57.83+11.49$ & $52.50+9.47$ & $0.00 * *$ \\
\hline \multicolumn{2}{|l|}{ Sex } & $163(75.5 \%)$ & 73 (75.3\%) & 90 (75.6\%) & 0.95 & 89 (74.2\%) & 74 (77.l\%) & 0.62 \\
\hline \multicolumn{2}{|c|}{ PTD (hour) } & $5.30+2.98$ & $6.30+2.69$ & $4.49+2.97$ & $0.00 * *$ & $6.05+2.85$ & $4.39+2.90$ & $0.00 * *$ \\
\hline \multicolumn{2}{|c|}{ HR (beat/min) } & $90.9+24.74$ & $106.76+23.94$ & $78.14+16.68$ & $0.00 * *$ & $102.90+22.92$ & $76.11+18.02$ & $0.00 * *$ \\
\hline \multicolumn{2}{|l|}{ SBP } & $|23.6|+30.29$ & $111.44+32.56$ & $133.53+24.27$ & $0.00^{* *}$ & || $6.88+3|.0|$ & $132.03+27.28$ & $0.00 * *$ \\
\hline \multicolumn{2}{|l|}{ DBP } & $75.04+17.99$ & $67.99+19.99$ & $80.79+13.79$ & $0.00 * *$ & $69.54+|8.4|$ & $81.93+14.89$ & $0.00 * *$ \\
\hline \multicolumn{2}{|c|}{ Cardiac enzymes } & $1170.82+1628.49$ & $1353.58+\mid 825.55$ & $1021.86+1439.06$ & 0.137 & || $88.45+|586.5|$ & $1148.79+1687.64$ & 0.859 \\
\hline \multicolumn{2}{|c|}{$\mathrm{EF}$} & $50.85+11.25$ & $49.81+13.10$ & $51.71+9.46$ & 0.22 & $48.88+12.26$ & $53.33+9.35$ & $0.004^{*}$ \\
\hline \multicolumn{2}{|l|}{ Smoking } & 91 (42.1\%) & 42 (43.3\%) & 49 (4I.2\%) & 0.75 & 50 (4l.7\%) & $4 \mathrm{I}(42.7 \%)$ & 0.87 \\
\hline \multicolumn{2}{|l|}{ DM } & 72 (33.3\%) & 37 (38.1\%) & 35 (29.4\%) & 0.17 & $43(35.8 \%)$ & $29(30.2 \%)$ & 0.38 \\
\hline \multicolumn{2}{|l|}{ HTN } & $84(38.9 \%)$ & 47 (48.5\%) & 37 (3I.I\%) & $0.009 *$ & 49 (40.8\%) & 35 (36.5\%) & 0.51 \\
\hline \multicolumn{2}{|c|}{ History of IHD } & 55 (25.5\%) & $26(26.8 \%)$ & $29(24.4 \%)$ & 0.68 & $32(26.7 \%)$ & $23(24.0 \%)$ & 0.65 \\
\hline \multirow{2}{*}{$\begin{array}{l}\text { Previous } \\
\text { procedure }\end{array}$} & $\mathrm{PCl}$ & $22(10.2 \%)$ & $8(8.2 \%)$ & I4 (II.8\%) & 0.29 & $13(10.8 \%)$ & $9(9.4 \%)$ & 0.27 \\
\hline & CABG & $2(0.9 \%)$ & $0(0.0 \%)$ & $2(1.7 \%)$ & & $0(0.0 \%)$ & $2(2.1 \%)$ & \\
\hline \multicolumn{2}{|c|}{ Positive family $\mathrm{H}$} & $35(16.2 \%)$ & $17(17.5 \%)$ & $18(15.1 \%)$ & 0.63 & $24(20.0 \%)$ & II (II.5\%) & 0.09 \\
\hline \multicolumn{2}{|c|}{ History of STROKE } & $6(2.8 \%)$ & $3(3.1 \%)$ & $3(2.5 \%)$ & 0.79 & $3(2.5 \%)$ & $3(3.1 \%)$ & 0.78 \\
\hline \multicolumn{2}{|c|}{ Pre-infarction angina } & $59(27.3 \%)$ & $34(35.1 \%)$ & $25(21.0 \%)$ & $0.02^{*}$ & $37(30.8 \%)$ & $22(22.9 \%)$ & 0.19 \\
\hline \multirow[t]{3}{*}{ Rhythm } & $\mathrm{AF}$ & $4(1.9 \%)$ & $2(2.1 \%)$ & $2(1.7 \%)$ & 0.96 & I (0.8\%) & $3(3.1 \%)$ & 0.45 \\
\hline & Paced & $2(0.9 \%)$ & I (I.0\%) & I (0.8\%) & & I $(0.8 \%)$ & I (I.0\%) & \\
\hline & SR & $210(97.2 \%)$ & 94 (96.9\%) & 116 (97.5\%) & & 118 (98.3\%) & $92(95.8 \%)$ & \\
\hline \multirow[t]{4}{*}{ Killip class } & 0 & I 72 (79.6\%) & $64(66.0 \%)$ & 108 (90.8\%) & $0.00 * *$ & 86 (71.7\%) & $86(89.6 \%)$ & $0.014^{*}$ \\
\hline & II & 24 (11.1\%) & $17(17.5 \%)$ & 7 (5.9\%) & & $19(15.8 \%)$ & $5(5.2 \%)$ & \\
\hline & III & II (5.1\%) & 7 (7.2\%) & $4(3.4 \%)$ & & $8(6.7 \%)$ & $3(3.1 \%)$ & \\
\hline & IV & $9(4.2 \%)$ & $9(9.3 \%)$ & $0(0.0 \%)$ & & $7(5.8 \%)$ & $2(2.1 \%)$ & \\
\hline \multicolumn{2}{|l|}{ Modified SI } & $1.03+0.45$ & $1.33+0.51$ & $0.79+0.15$ & $0.00 * *$ & & & \\
\hline \multicolumn{2}{|l|}{ ASI } & $0.77+0.33$ & & & & $0.93+0.33$ & $0.59+0.23$ & $0.00 * *$ \\
\hline \multicolumn{2}{|l|}{ TIMlrs } & $4.1+2.37$ & $6.15+1.53$ & $2.45+1.46$ & $0.00 * *$ & $5.35+1.99$ & $2.56+1.85$ & $0.00 * *$ \\
\hline
\end{tabular}

AF: Atrial Fibrillation;ASI:Admission Shock Index; CABG: Coronary Artery Bypass Graft; DM: Diabetes Mellitus; DBP: Diastolic Blood Pressure; F: Female; HR: Heart Rate; HTN: Hypertension; IHD: Ischemic Heart Disease; M: Male; MSI: Modified Admission Shock Index; PCI: Percutaneous Coronary Intervention; PTD: Pain to Door Time; SBP: Systolic Blood Pressure; SR: Sinus Rhythm.

Table 2 The in-hospital outcomes stratified by ASI, MSI, and TIMIrs

\begin{tabular}{|c|c|c|c|c|c|c|c|c|c|c|}
\hline Outcome & $\begin{array}{l}\text { All Patients } \\
(n=216)\end{array}$ & $\begin{array}{l}\text { ASI }>0.69 \\
(n=97)\end{array}$ & $\begin{array}{l}A S \mid<0.69 \\
(n=I \mid 9)\end{array}$ & P-value & $\begin{array}{l}\text { MSI > 0.9I } \\
(n=\mid 20)\end{array}$ & $\begin{array}{l}M S I<0.9 I \\
(n=96)\end{array}$ & P-value & $\begin{array}{l}\text { TIMI > } 4 \\
(n=102)\end{array}$ & $\begin{array}{l}\text { TIMI <4 } \\
(n=I \mid 4)\end{array}$ & P-value \\
\hline $\mathrm{HF}$ & $38(17.6 \%)$ & $26(26.8 \%)$ & $12(10.1 \%)$ & $0.001 * *$ & $32(26.7 \%)$ & $6(6.2 \%)$ & $0.00 * *$ & $32(26.4 \%)$ & $6(6.3 \%)$ & $0.000 * *$ \\
\hline CS & 14 (6.5\%) & $12(12.4 \%)$ & $2(1.7 \%)$ & $0.002 *$ & $12(10.0 \%)$ & $2(2.1 \%)$ & $0.019 *$ & 12 (9.9\%) & $2(2.1 \%)$ & $0.014^{*}$ \\
\hline Re-Infarction & I (0.5\%) & I (I.0\%) & $0(0.0 \%)$ & 0.26 & I (0.8\%) & $0(0.0 \%)$ & 0.37 & I (0.8\%) & $0(0.0 \%)$ & $0.28 I$ \\
\hline $\begin{array}{l}\text { Fatal } \\
\text { Arrhythmia }\end{array}$ & I7 (7.9\%) & 12 (I2.4\%) & $5(4.2 \%)$ & $0.027^{*}$ & $13(10.8 \%)$ & $4(4.2 \%)$ & $0.007^{*}$ & $13(10.7 \%)$ & $4(4.2 \%)$ & 0.068 \\
\hline Bleeding & $6(2.8 \%)$ & $4(4.1 \%)$ & $2(1.7 \%)$ & 0.27 & $6(5.0 \%)$ & $0(0.0 \%)$ & $0.026 *$ & $6(5.0 \%)$ & $0(0.0 \%)$ & $0.008^{*}$ \\
\hline Stroke & $2(0.9 \%)$ & $2(2.1 \%)$ & $0(0.0 \%)$ & 0.11 & $2(1.7 \%)$ & $0(0.0 \%)$ & 0.2 & 2 (1.7\%) & $0(0.0 \%)$ & 0.127 \\
\hline Arrest & 32 (I4.8\%) & 25 (25.8\%) & 7 (5.9\%) & $0.00 * *$ & 25 (20.8\%) & 7 (7.3\%) & $0.005^{*}$ & $26(21.5 \%)$ & $6(6.3 \%)$ & $0.001 *$ \\
\hline Mortality & $19(8.8 \%)$ & $16(16.5 \%)$ & $3(2.5 \%)$ & $0.00 * *$ & $16(13.3 \%)$ & $3(3.1 \%)$ & $0.008^{*}$ & $16(13.2 \%)$ & $3(3.2 \%)$ & $0.006 *$ \\
\hline
\end{tabular}

ASI:Admission Shock Index; CS: Cardiogenic Shock; HF: Heart Failure; MSI: Modified Admission Shock Index;TIMI:Thrombolysis in Myocardial Infarction Risk Score.

Citation: Gouda M, Saad AM,Al- Daydamony MM. Modified shock index as a predictor of in-hospital outcome in cases of st-segment elevation myocardial infarction treated with primary percutaneous coronary intervention.J Cardiol Curr Res. 2016;7(4):I-6. DOI: I0.15406/jccr.20I6.07.00255 
Table 3 Predictors of in-hospital mortality and MACE by multivariate Cox analysis

\begin{tabular}{|c|c|c|c|c|c|c|c|}
\hline \multicolumn{4}{|l|}{ Mortality } & \multicolumn{4}{|l|}{ MACE } \\
\hline Variable & ORs & 95\% C.I. & P-value & Variable & ORs & 95\% C.I. & P-value \\
\hline History of IHD & 0 & 0 & 0.999 & Killip classification & $\mathrm{I} .144$ & $0.681-1.922$ & 0.611 \\
\hline Killip classification & 0.983 & $0.419-2.302$ & 0.968 & LAD & 1.813 & $0.695-4.731$ & 0.224 \\
\hline Age & 1.079 & $1.009-1.153$ & $0.025^{*}$ & $\mathrm{HR}$ & I & $0.967-1.033$ & 0.989 \\
\hline chest pain duration & 1.066 & $0.807-1.407$ & 0.652 & SBP & 1.002 & $0.979-1.027$ & 0.853 \\
\hline $\mathrm{HR}$ & $0.94 I$ & $0.845-1.047$ & 0.266 & $\mathrm{EF}$ & 0.879 & $0.840-0.921$ & $0.000 *$ \\
\hline SBP & 1.056 & $0.977-1 .|4|$ & 0.172 & ASI & 1.21 & $1.05-3.54$ & $0.012 *$ \\
\hline EF & 0.822 & $0.74|-0.9| I$ & $0.000 *$ & MSI & II.506 & $10.54-21.5664$ & $0.022 *$ \\
\hline ASI & 2.87 & $1.08-3.484$ & $0.04 *$ & TIMI & 1.575 & $1.094-2.269$ & $0.015^{*}$ \\
\hline MSI & 5.599 & $4.128-8.15$ & $0.015^{*}$ & Constant & 33.711 & & 0.114 \\
\hline TIMI & 1.238 & I.II -2.87 & $0.021 *$ & & & & \\
\hline Constant & 0.002 & & 0.269 & & & & \\
\hline
\end{tabular}

ASI:Admission Shock Index; SBP: Systolic Blood Pressure; EF: Ejection Fraction; HR: Heart Rate; IHD: Ischemic Heart Disease; LAD: Left Anterior Descending Artery; MSI: Modified Admission Shock Index;TIMIrs:Thrombolysis in Myocardial Infarction Risk Score.

Table 4 Correlations between studied parameters (ASI, MSI and TIMIrs)

\begin{tabular}{|c|c|c|c|c|c|c|c|}
\hline \multicolumn{8}{|l|}{ Correlations } \\
\hline & & Age & Weight & EF & ASI & Modified SI & TIMIRS \\
\hline \multirow[t]{3}{*}{ ASI } & $r$ & 0.036 & 0.018 & $-.170-*$ & 1 & $.829 * *$ & $.681 * *$ \\
\hline & $\mathrm{P}$ & 0.599 & 0.788 & 0.012 & & 0 & 0 \\
\hline & $\mathrm{N}$ & 216 & 216 & 216 & 216 & 216 & 216 \\
\hline \multirow[t]{3}{*}{ Modified SI } & $r$ & 0.068 & 0.009 & $-.188-* *$ & $.829 * *$ & I & $.579 * *$ \\
\hline & $P$ & 0.323 & 0.899 & 0.006 & 0 & & 0 \\
\hline & $\mathrm{N}$ & 216 & 216 & 216 & 216 & 216 & 216 \\
\hline \multirow[t]{3}{*}{ TIMIRS } & $r$ & $.27 I^{* *}$ & $-.024-$ & $-.215-* *$ & $.681 * *$ & $.579 * *$ & 1 \\
\hline & $P$ & 0 & 0.723 & 0.001 & 0 & 0 & \\
\hline & $\mathrm{N}$ & 216 & 216 & 216 & 216 & 216 & 216 \\
\hline \multicolumn{8}{|c|}{ **. Correlation is significant at the 0.01 level (2-tailed). } \\
\hline \multicolumn{8}{|c|}{ *. Correlation is significant at the 0.05 level (2-tailed). } \\
\hline
\end{tabular}

EF: Ejection Fraction;TIMIrs:Thrombolysis in Myocardial Infarction Risk Score;ASI:Admission Shock Index; MSI: Modified Admission Shock Index.
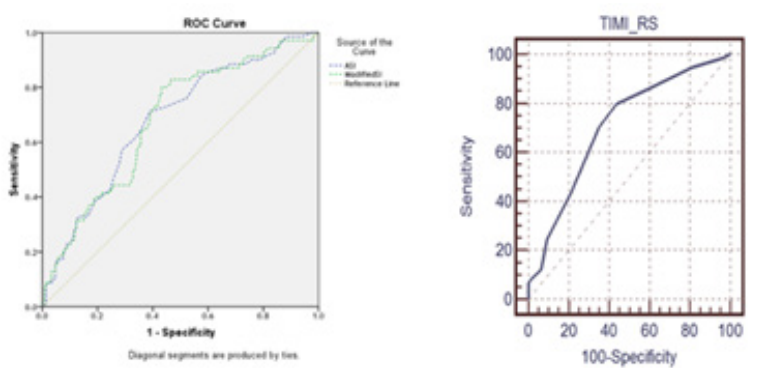

Figure I Shows ROC curve for ASI, MSI and TIMIrs among all studied cases.

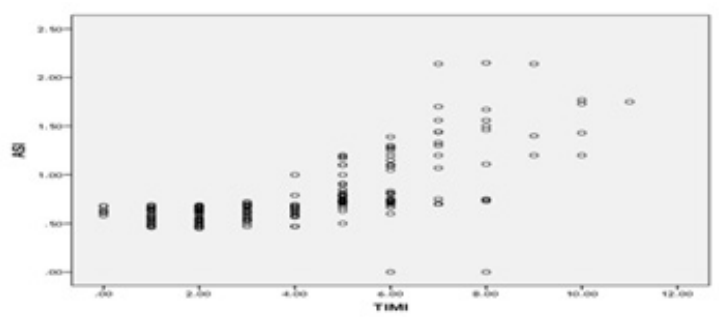

Figure 2 Shows positive correlation between ASI and TIMI risk score.

\section{Discussion}

Cardiogenic shock (CS) remains the most common cause of death in STEMI patients. ${ }^{12}$ Although CS incidence has declined with PPCI, ${ }^{13}$ it still occurs in $5-8 \%$ with approximately $50 \%$ mortality. ${ }^{12}$
In the setting of STEMI especially complicated by cardiogenic shock, parameters related to cardiac function such as cardiac index, stroke volume, and left ventricular stroke work decrease and a series of neurohumoral reactions are aroused after myocardial infarction, of which sympathetic nerve activation is the most significant. ${ }^{14}$ Associating with increased catecholamine release promoted elevation of $\mathrm{BP}$ and HR occurs to compensate lowered cardiac output due to myocardial infarction. ${ }^{15}$ Therefore, BP and HR index after myocardial infarction may be a reflection of integrated cardiovascular system and neuroendocrine system as well as hemodynamics status. Indeed, several risk scores have recognized the importance of BP and HR and have taken the 2 variables into the risk score models..$^{5-7}$ In fact, as an integrated index of the 2 variables, SI is a sensitive indicator of left ventricular dysfunction and has been shown to be a better measure of the degree of hemodynamic stability than HR or SBP alone. ${ }^{16}$ Furthermore, even in the presence of normal HR and BP, SI has been demonstrated to be a useful marker of early acute hypovolemia, ${ }^{17}$ and a persistent elevation of the SI for several hours would significantly increase the morbidity and mortality in acute circulatory failure. ${ }^{10}$ Moreover, SI is mostly independent of pain and anxiety, ${ }^{18}$ which cause a concurrent increase in HR and SBP, and therefore, was a relatively objective indicator reflecting the disease state. These results taken together suggest that the predictive value of admission SI for short-term outcomes in patients with STEMI may be mediated by the recognition of hemodynamic alteration sensitively. The simple algorithm also makes its use accessible for risk stratification during the first contact with patients. 


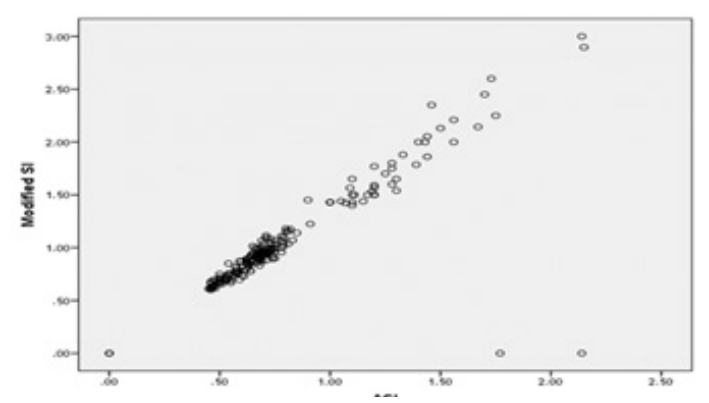

Figure 3 Shows positive correlation between MSI and TIMI risk score.

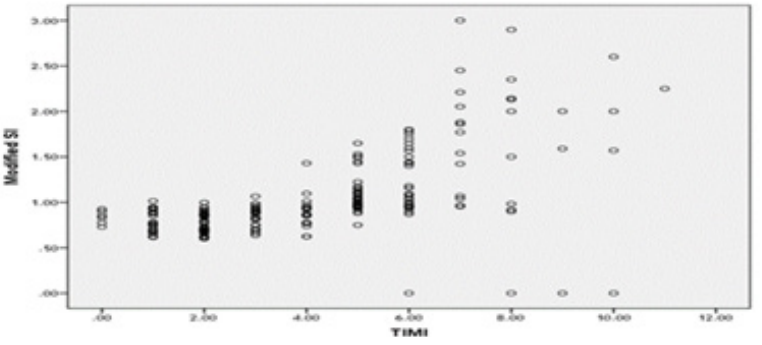

Figure 4 Shows positive correlation between ASI and MSI.

In patients with established CS, complete revascularization is recommended. ${ }^{19}$ However, no study or recommendation has suggested any benefit from such an approach in patients at risk of developing $\mathrm{CS}$ and this may be an area for future studies. A high MSI denotes a value of stroke volume and low systemic vascular resistance, a sign of hypodynamic circulation. Thus the patient may be compensating and the decompensation is rapid. Given the limited resources and information available at triage, MSI can be a valuable tool in predicting disease severity. Studies results show that in patients with a high MSI, there is an increased probability of ICU admission and death.MSI is a more important clinical predictor than blood pressure and heart rate because high MSI indicates a hypodynamic state. ${ }^{11}$

In our study, elevated SI was associated with a significant increase of incidence of cardiogenic shock, fatal arrhythmia, and with a highly significant increase of incidence of HF, arrest and mortality. That was in agreement with Bi Huang et al., ${ }^{20}$ who showed if ASI $>0.7$, there was a highly significant increase of incidence of 7-day HF, cardiogenic shock, fatal arrhythmia, arrest, and mortality. ${ }^{20}$ While in the study done by Qing Shangguan et al., ${ }^{21}$ with ASI $>0.7$ there was a significant increase of incidence of 7-day MACE, Killip class, fatal arrhythmia, and all-cause mortality, but they found no significant increase in incidence of cardiogenic shock. ${ }^{21}$ Dana Bilkova et al., ${ }^{22}$ used a SI cut off $=0.8$, they worked on evaluating the relation between SI and the inhospital mortality, they showed that with ASI $>0.8$, there was a highly significant increase of incidence of in-hospital mortality. ${ }^{22}$ Lastly in a study done by Ioakim Spyridopoulos et al., ${ }^{23}$ that enrolled 3049 consecutive STEMI patient treated by PPCI prospectively and with SI cut off $=1.0$, showed that with ASI $>1.0$ there was a highly significant increase of incidence of in-hospital mortality. ${ }^{23}$

In our study, elevated MSI was associated with a significant increase of incidence of cardiogenic shock, fatal arrhythmia, bleeding, arrest and mortality. This was associated with a highly significant increase of incidence of HF. That was in agreement with results of Qing Shangguan et al., ${ }^{21}$ who showed that with MSI $>1.4$, there was a highly significant increase of incidence of 7-day MACE, fatal arrhythmia, and all-cause mortality, and there was a significant increase of incidence of Killip class, but he found no significant increase in incidence of cardiogenic shock. ${ }^{21}$ In another recent study done by Gloria Abreu et al., ${ }^{24}$ who enrolled 1140 consecutive STEMI patients treated by both types of reperfusion (pharmacological and mechanical) retrospectively, and with MSI cut off $=1.3$ showed that with MSI $>1.3$ there was a significant increase of incidence of inhospital mechanical complications, malignant arrhythmia, and there was a highly significant increase of incidence of respiratory tract infections, and all-cause mortality. ${ }^{24}$

\section{According to relation between TIMI and MACE}

In our study, high TIMIrs with cutoff value of 4 was associated with highly statistically significant increase in HF, statistically significant increase in cardiogenic shock, non-fatal arrhythmia, bleeding, arrest and mortality. This was in agreement with results of a most recent study done by Halit Acet et al., who enrolled 390 consecutive STEMI patient treated by PPCI retrospectively. In another study done by Hector Gonzalez et al., ${ }^{25}$ who enrolled 572 consecutive STEMI patients treated by emergency PCI prospectively, showed that with TIMI $>5$ there was a highly significant increase of incidence of inhospital mortality, cardiogenic shock, ventricular arrhythmia, and HF, But he found no significant increase in incidence of re-infarction. ${ }^{25}$

\section{According to the correlation between TIMIrs, ASI, and MSI}

Our study found that, there is highly significant positive correlation between ASI, modified SI and TIMIrs and also between TIMIrs and age modified SI but significant negative correlation between EF ASI, TIMI and Modified SI. To the best of our knowledge, no previous study worked on the correlation study between these three parameters (ASI, MSI, and TIMIrs).

For mortality, in our study MSI was the strongest predictor of in-hospital mortality. Other predictors were ASI, TIMIrs, age, and EF. For MACE, in our study, MSI was the strongest predictor of inhospital MACE then ASI, TIMIrs, and EF. That was in agreement with Qing Shangguan et al., ${ }^{21}$ where multifactor analysis showed that, in addition to MSI or ASI, age was independent factor for the 7-day MACE, with the OR of MSI was higher than that of age (3.05 vs 1.07). These results indicated that the poorer outcome in the increased MSI groups is not because of the older age, as the older patients may have higher SBP and lower DBP and then had higher MSI. Thus, MSI per se is a useful predictor. The analysis also found that blood glucose level was another independent factor for the 7-day MACE.

\section{Finally our study findings are:}

At first, the present study demonstrated that SI $\geq 0.69$, and MSI $\geq 0.91$ are useful predictors for in-hospital mortality and MACE in the patients with STEMI treated by PPCI.

Increased mortality associated with elevated SI and MSI were in part explained by the higher incidence of cardiogenic shock. In our study, patients with elevated SI had a higher rate of cardiogenic shock on admission. In addition, patients with elevated SI were more likely to develop cardiogenic shock after admission, suggesting that elevated SI can be an early sign of impending hemodynamic instability leading to cardiogenic shock, especially when we evaluated the mortality of patients with a systolic blood pressure $<90 \mathrm{~mm} \mathrm{Hg}$ and an SI $<0.69$, the mortality rate was zero. However, it is necessary to note that this group was extremely small (3 patients).

The present study demonstrated that MSI $(\geq 0.91)$ is a better predictor than SI $(\geq 0.69)$ for in-hospital all-cause mortality and MACE in the patients with STEMI treated with PPCI. 
The present study for the first time demonstrated that although MSI is more simple than multifactorial TIMI risk score, but MSI $(\geq 0.91)$ is a better predictor than TIMIrs for in-hospital all-cause mortality and MACE in the patients with STEMI treated with PPCI.

\section{Study limitations}

The current study had the following limitations:

a. The sample size was rather small.

b. The follow-up time was short-term.

c. The population included was only admitted to a single center.

d. We evaluated ASI in only one type of ACS, which is STEMI, and with only one way for reperfusion, which is PPCI.

\section{Conclusion}

Both SI and MSI measured in ED could predict the in-hospital allcause mortality and MACE in patients with STEMI treated by PPCI. MSI is stronger independent factor for predicting in-hospital mortality and MACE than TIMIrs. The predictors of in-hospital mortality were MSI, ASI, TIMIrs, age, and EF. While predictor of in-hospital MACE were MSI, ASI, TIMIrs and EF.

\section{Acknowledgments}

None.

\section{Conflicts of interest}

Author declares there is no conflicts opf interest.

\section{Funding}

None.

\section{References}

1. Laslett LJ, Alagona P, Clark BA, et al. The worldwide environment of cardiovascular disease: Prevalence, diagnosis, therapy, and policy issues: A report from the American College of Cardiology. $J$ Am Coll Cardiol. 2012;60(25):S1-S49.

2. Lenzen MJ, Boersma E, Bertrand ME, et al. Management and outcome of patients with established coronary artery disease: the Euro Heart Survey on coronary revascularization. Eur Heart J. 2005;26(12):1169-1179.

3. Stephan W, Philippe K. Task Force on Myocardial Revascularization of the European Society of Cardiology (ESC) and the European Association for Cardio-Thoracic Surgery (EACTS) Developed with the special contribution of the European Association of Percutaneous Cardiovascular Interventions (EAPCI). European Heart Journal. 2014;35:2541-2619.

4. O'Gara PT, Kushner FG, Ascheim DD, et al. 2013 ACCF/AHA guideline for the management of ST-elevation myocardial infarction: a report of the American College of Cardiology Foundation/American Heart Association Task Force on Practice Guidelines. Circulation. 2013;127(4):e362-e425.

5. Morrow DA, Antman EM, Charlesworth A, et al. TIMI risk score for ST-elevation myocardial infarction: a convenient, bedside, clinical score for risk assessment at presentation: an intravenous nPA for treatment of infracting myocardium early II trial sub-study. Circulation. 2000;102(17):2031-2037.

6. Granger CB, Goldberg RJ, Dabbous O, et al. Predictors of hospital mortality in the global registry of acute coronary events. Arch Intern Med . 2003;163(19):2345-2353.
7. Halkin A, Singh M, Nikolsky E, et al. Prediction of mortality after primary percutaneous coronary intervention for acute myocardial infarction: the CADILLAC risk score. J Am Coll Cardiol . 2005;45(9):1397-1405.

8. De Mulder Maarten, Aly Saad, Anselm Gitt, et al. EuroHeart score for the evaluation of in-hospital mortality in patients undergoing percutaneous coronary intervention. Eur Heart J. 2011;32:1398-1408.

9. Allgower M, Burri C. Shock index. Dtsch Med Wochenschr. 1967;92(43):1947-1950.

10. Rady MY, Nightingale P, Little RA, et al. Shock index: a re-evaluation in acute circulatory failure. Resuscitation. 1992;23(3):227-234.

11. Liu YC, Liu JH, Fang ZA, et al. Modified shock index and mortality rate of emergency patients. World J Emerg Med. 2012;3(2):114-117.

12. Reynolds HR, Hochman JS. Cardiogenic shock: Current concepts and improving outcomes. Circulation . 2008;117(5):686-697.

13. Babaev A, Frederick PD, Pasta DJ, et al. Trends in management and outcomes of patients with acute myocardial infarction complicated by cardiogenic shock. JAMA. 2005;294(4):448-454.

14. Graham LN, Smith PA, Stoker JB, et al. Sympathetic neural hyperactivity and its normalization following unstable angina and acute myocardial infarction. Clin Sci (Lond). 2004;106:605-611.

15. Ceremuzynski L. Hormonal and metabolic reactions evoked by acute myocardial infarction. Circ Res. 1981;48(6 Pt 1):767-776.

16. Zarzaur BL, Croce MA, Fischer PE, et al. New vitals after injury: shock index for the young and age $\mathrm{x}$ shock index for the old. $J$ Surg Res 2008;147(2):229-236.

17. Rady MY, Rivers EP, Martin GB, et al. Continuous central venous oximetry and shock index in the emergency department: use in the evaluation of clinical shock. Am J Emerg Med. 1992;10(6):538-541.

18. Keller AS, Kirkland LL, Rajasekaran SY, et al. Unplanned transfers to the intensive care unit: the role of the shock index. J Hosp Med. 2010;5(8):460-465.

19. Hochman JS, Sleeper LA, Webb JG, et al. for the SHOCK Investigators: Early revascularization in acute myocardial infarction complicated by cardiogenic shock. SHOCK Investiga-tors. Should We Emergently Revascularize Occluded Coronaries for Cardiogenic Shock? N Engl J Med. 1999;341(9):625-634.

20. Huang B, Yang Y, Zhu J, et al. Usefulness of the Admission Shock Index for Predicting Short-Term Outcomes in Patients With ST-Segment Elevation Myocardial Infarction. Am J Cardiol . 2014;114(9):1315-1321.

21. Shangguan Q, Xu JS, Su H, et al. Modified shock index is a predictor for 7-day outcomes in patients with ST-segment elevation myocardial infarction. Am J Emerg Med . 2015;33(8):1072-1075.

22. Bilkova D, MotovskaZ, Widimsky P, et al. Shock Index: A Simple Clinical Parameter for Quick Mortality Risk Assessment in Acute Myocardial Infarction, Canadian Journal of Cardiology . 2011;27(6):739-742.

23. Spyridopoulos I, Noman A, Ahmed JM, et al. Shock-index as a novel predictor of long-term outcome following primary percutaneous coronary intervention. Eur Heart J Acute Cardiovasc Care. 2014;4(3):270-277.

24. Glória Abreu, Carlos Braga, Carina Arantes. Modified shock index -a strong predictor of outcome among patients presenting ST-segment elevation myocardial infarction. World JEmerg Med. 2012;3(2):114-117.

25. Héctor González-Pacheco, Alexandra Arias-Mendoza, Amada ÁlvarezSangabriel, et al. The TIMI risk score for STEMI predicts in-hospital mortality and adverse events in patients without cardiogenic shock undergoing primary angioplasty. Arch Cardiol Mex. 2012;82(1):7-13. 